\title{
ON PURPOSEFULNESS OF APPLICATION OF PHYSICAL CULTURE-HEALTH RELATED TECHNOLOGY FOR PROPHYLAXIS OF STUDENTS' RESPIRATORY SYSTEM DISORDERS
}

Kondakov V.L., Kopeikina E.N., Usatov A.N.

National Research University Belgorod State University

\begin{abstract}
Purpose: To give scientific foundation and experimental proof of physical culture-health related technology for prophylaxis of respiratory system disorders. Material: in the research students: girls $(\mathrm{n}=43)$ and boys $(n=40)$ participated. In process of academic physical culture classes students practices: dozed health walks ) as warming up), respiratory exercises by methodic of A.N. Strelnikova and G. Childers (which were used as main corrective mean of respiratory system's functional state), health swimming (for motor functioning intensification) outdoor games (as mean of active leisure). Results: The conducted research witness about general positive influence of the worked out technology of disorders' prophylaxis on students' somatic health. The technology was constructed on the base of synthesis of the most effective means of health related physical culture. In its basis we put generalized data about their impact. Main characteristic of this technology is its orientation on definite health improvement tasks in compliance with peculiarities of trainees' contingent. The technology permits to present its content and orientation as integral mean of strengthening of organism's functional potentials as well as ensuring of high effectiveness of students' educational process. It permits to significantly reduce impacts of respiratory system's disorders. Conclusions: The worked out technology permits to present its content and orientation as integral mean of strengthening of organism's functional potentials.
\end{abstract}

Key words: physical culture-health related technology, motor functioning, respiratory exercises, students.

\section{Introduction}

Recent years academic programs in Russia Federation higher educational establishments have significantly increased in volume and complicated in content. It resulted in noticeable increase of independent work and nearly in $50 \%$ of motor functioning reduction $[1,2$, and 3$]$. We can state that about half of modern students of Russian HEEs have health problems [1, 2, 3, 4, 5, 6, 7, and 19].

The conducted by us theoretical analysis and generalization of literature data on problem of prophylaxis of students' respiratory system disorders proves the presence of problem situation [15, 20, 21, 22, 23].

Experimental material on this problem, accumulated up to the present time, witnesses that students' everyday motor functioning does not ensure optimal development of organism's main physiological functions and does not create conditions for health strengthening. It was found that with every year quantity of students with health problems increases. Quantity of students, whom it is prohibited by medicine to practice physical culture, also increases [2, 5, and 19].

In the basis of scientific foundation of physical culture-health related technology's content and orientation there is prophylaxis of respiratory system disorders. The technology contains criteria, formulated by us earlier in development of federal targeted program "Scientific and scientific-pedagogic personnel of innovative Russia" for 2009-2013 [10]:

- Content of physical culture-health related technology shall render complex health improvement influence; facilitate perfection of external breathing functions, increase of vital capacity of lungs, mobility of chest and lungs, recreation of air tracts' pass ability, coordination of breathing act;

- Mean and methods of physical culture-health related technology shall facilitate recreation of physical and mental workability, release nervous-emotional tension;

- In the base of motor functioning content exercises with regulated physical loads' intensity shall be embedded;

- Physical exercises shall exclude possibility of traumas and be easy in fulfillment [1, 2, 3, 4, 5, and 18].

The conducted profound analytical researches showed that the most popular kinds of motor functioning are: health run, combined with walking [8,21], athletic gymnastic $[9,10]$, swimming $[14,16]$, sports and outdoor games $[8,15]$. In groups of students, who practiced breathing exercises, positive influence on respiratory system was registered [8, 16, 17, 18, and 19]. All mentioned kinds of motor activity should be used as additional means at trainings of students with respiratory system disorders.

Purpose, tasks of the work, material and methods

The purpose of the work is to give scientific foundation and experimental proof of physical culture-health related technology for prophylaxis of respiratory system disorders. Such technology can be constructed on the base of synthesis of the most effective means of health related physical culture. In its basis we put generalized data about their impact in educational space of modern HEE.

(C) Kondakov V.L., Kopeikina E.N., Usatov A.N., 2016

doi:10.15561/20755279.2016.0201 
As main means of technology for prophylaxis of respiratory system we used the following:

$\checkmark \quad$ Dozed health walks was applied in preparation part of training as warming up); in independent trainings it was used as mean of motor functioning increase as well as students' psycho-physiological fitness);

$\checkmark \quad$ Respiratory exercises by methodic of A.N. Strelnikova and G. Childers (which were used as main corrective mean of respiratory system's functional state; in independent trainings it was used as mean of motor functioning increase as well as students' psycho-physiological fitness);

- Health swimming (was used in independent trainings as mean for increasing of students' motor functioning);

- Outdoor and sports games, relays (were used in main part of training session as mean of active rest for releasing tension, rising mood, activity and students' psycho-physiological fitness);

- Exercises for basic physical qualities - complex training (was practiced in main part of training session, considering counter indications for students with respiratory system problems, for rising students' physical and psycho-physiological fitness);

- General developing exercises (were used in warming up part, considering recommendations for persons with respiratory system problems, for preparation of students' organism for following loads);

- Stretching and relaxation exercises (were used in final part of training for recreation students' organism after loads).

On organizational stage we formed the groups of tested: two experimental (EGg - girls and EGb- boys) and two control groups (CGg - girls and CGb - boys).

Pilot testing of experimental technology envisaged 12 curricular physical culture classes. The trainings were conducted twice a week without extracurricular trainings. Control groups were trained by program, approved by physical education department. This program was worked out in compliance with requirements of state program for HEEs on discipline "physical culture".

Pilot experiment had purpose to determine effectiveness of the worked out physical culture-health related technology for prophylaxis of respiratory system disorders effectiveness in conditions of HEE educational space. The experiment was carried out with students of main department, who had no expressed health problems. The physical culture-health related technology was tested by two groups; group of boys and group of girls.

All participants of experiment were familiarized with "Helsinki declaration of world health protection organization" and gave written consent for participation in experiment [11].

Statistical analysis

Statistical analysis of the received data was fulfilled with the help of licensed electronic tables Excel (2010). We determined the following indicators of descriptive statistic: mean arithmetic value, standard deviation and error of mean arithmetic) confidence of differences was assessed by Student's criterion. The difference considered to be confident at $\mathrm{p}<0.05$.

\section{Results of the research}

The received by us data witness that physical condition was not noticeably influenced by experimental physical culture-health related technology. Alongside with it, positive changes in boys groups by indicators of chest excursion and vital capacity of lungs should be noted. These indicators are important characteristics of respiratory system condition (see tables 1,2 ).

Table 1. Dynamic of students' indicators in corrected variant of physical culture-health related technology for prophylaxis of respiratory disorders (girls)

\begin{tabular}{|c|c|c|c|c|c|c|c|c|c|c|c|}
\hline \multirow{3}{*}{ Indicators } & \multicolumn{7}{|l|}{ EG } & \multicolumn{4}{|l|}{ CG } \\
\hline & \multicolumn{3}{|c|}{$\begin{array}{l}\text { Before } \\
\text { experiment }\end{array}$} & \multicolumn{3}{|c|}{$\begin{array}{l}\text { After } \\
\text { experiment }\end{array}$} & \multirow[t]{2}{*}{$\mathbf{P}$} & \multicolumn{2}{|c|}{$\begin{array}{l}\text { Before } \\
\text { experiment }\end{array}$} & \multicolumn{2}{|c|}{$\begin{array}{l}\text { After } \\
\text { experiment }\end{array}$} \\
\hline & $\mathbf{M}$ & & m & $\mathbf{M}$ & \pm & m & & $\mathbf{M}$ & $\pm m$ & $\mathbf{M}$ & $\pm m$ \\
\hline \multicolumn{12}{|c|}{ Physical condition } \\
\hline Height (cm) & $\begin{array}{l}164.1 \\
5\end{array}$ & + & 2.54 & $\begin{array}{l}163.9 \\
0\end{array}$ & & 2.56 & & $\begin{array}{l}164 . \\
92\end{array}$ & \pm 0.24 & $\begin{array}{l}165 . \\
25\end{array}$ & \pm 0.86 \\
\hline Weight (kg) & 59.21 & \pm & 2.81 & 59.08 & \pm & 2.68 & & $\begin{array}{l}56.4 \\
3\end{array}$ & \pm 0.45 & $\begin{array}{l}55.2 \\
9\end{array}$ & \pm 0.39 \\
\hline Kettle's index (conv.un.) & 18.11 & \pm & 0.78 & 18.10 & \pm & 0.74 & & $\begin{array}{l}67.3 \\
0\end{array}$ & \pm 0.27 & $\begin{array}{l}66.9 \\
4\end{array}$ & \pm 0.96 \\
\hline Waist circumference $(\mathrm{cm})$ & 70.60 & \pm & 2.70 & 69.70 & \pm & 2.27 & & $\begin{array}{l}95.4 \\
4\end{array}$ & \pm 1.17 & $\begin{array}{l}95.1 \\
8\end{array}$ & \pm 1.23 \\
\hline
\end{tabular}




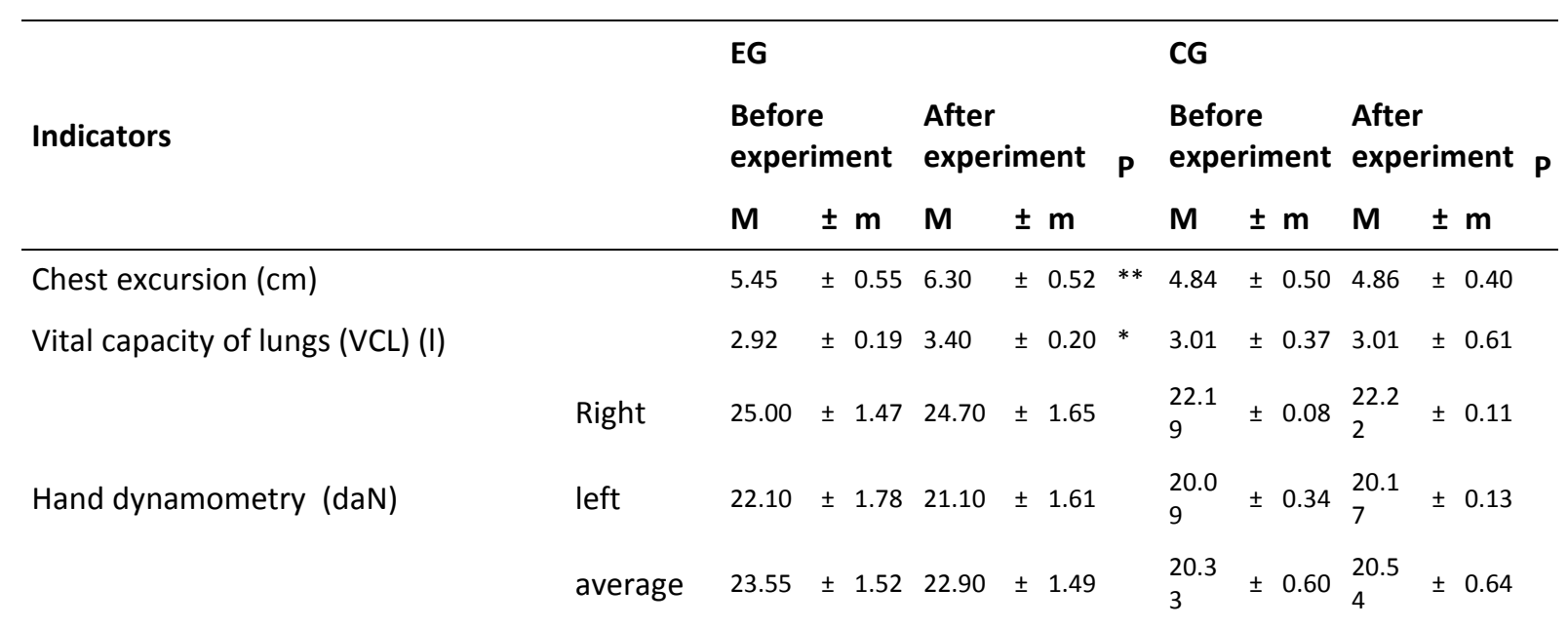

\section{Physical fitness}

Pressing ups in lying position (q-ty of times)

$151 \pm 0.724 .11 \pm 1.72 \quad 9.98 \pm 0.469 .88 \pm 0.57$

Torso rising from lying position (q-ty of times)

$35.31 \pm 3.6644 .81 \pm 3.76 * \begin{aligned} & 54.3 \\ & 2\end{aligned} \pm 1.02 \begin{aligned} & 55.0 \\ & 2\end{aligned} \pm 0.81$

Keeping of half squat with support (sec)

$76.92 \pm 9.8777, .6 \pm 8.44 \quad \begin{aligned} & 63.2 \\ & 4\end{aligned} \pm 6.60 \int_{4}^{62.7} \pm 2.33$

Long jump from the spot $(\mathrm{cm})$

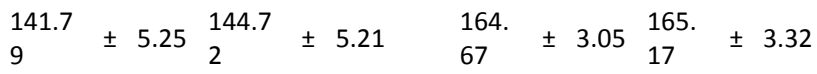

60 meters' run, sec.

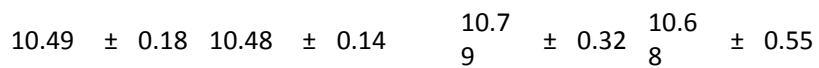

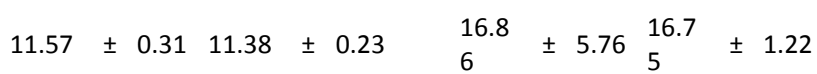

10 squatting for quickness, sec.

$\begin{aligned} & 1031 . \\ & 12\end{aligned} \pm \begin{array}{ll}20.8 & 1012 . \\ 4 & 53\end{array} \pm \begin{aligned} & 34.6 \\ & 2\end{aligned} \quad \begin{aligned} & 698 . \\ & 41\end{aligned} \quad \begin{aligned} & 30.1 \\ & 3\end{aligned}$

for boys, sec.

$2.81 \pm 3.558 .11 \pm 2.54 * \begin{aligned} & 10.2 \\ & 6\end{aligned} \pm 0.03 \begin{aligned} & 10.2 \\ & 2\end{aligned} \pm 0.53$

Bending from bench, $\mathrm{cm}$.

$5.66 \pm 1.235 .54 \pm 1.23 \quad 4.44 \pm 0.074 .48 \pm 0.18$

Firiliova's test (conv.un.)

Yarotskiy's test, sec.

$5.73 \pm 1.725 .83 \pm 1.67 \quad 6.29 \pm 0.596 .33 \pm 0.75$

Swimming, sec.

$74.56 \pm 2.0765 .20 \pm 1.91 \begin{array}{ll}* * & 67.1 \\ * & 1\end{array} \pm 1.48 \begin{aligned} & 67.0 \\ & 1\end{aligned} \pm 1.20$

\section{Functional fitness}

Heart beats rate (HBR) in rest, bpm

Blood pressure (mm.merc.col.)

Systolic blood volume (SBV) (ml)

Minute blood volumes (MBV) (I)

Shtange's test, sec.

Genchy's test, sec.

Orthostatic test (difference bpm)
$85.10 \pm 3.3580 .30 \pm 2.53 \quad 6^{76.3} \pm 9.86 \begin{aligned} & 75.4 \\ & 0\end{aligned} \pm 5.59$

Systolic $\begin{aligned} & 110.2 \\ & 0\end{aligned} \pm 2.74 \begin{aligned} & 106.6 \\ & 0\end{aligned} \pm 2.78 \quad \begin{aligned} & 114 . \\ & 23\end{aligned} \pm 0.19 \begin{aligned} & 114 . \\ & 80\end{aligned} \pm 0.77$

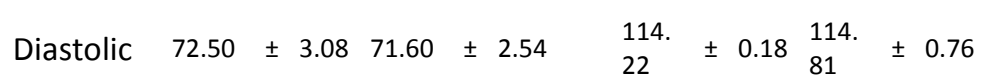

$58.87 \pm 3.1857 .92 \pm 3.06 \quad \begin{aligned} & 61.2 \\ & 0\end{aligned} \pm 0.59 \begin{aligned} & 60.9 \\ & 1\end{aligned} \pm 1.77$

$5.14 \pm 0.68 \quad 4.57 \quad \pm 0.61 \quad 4.67 \quad \pm 0.56 \quad 4.68 \pm 0.68$

$57.01 \pm 4.8172 .04 \pm 4.68 \begin{array}{ll}* * & 41.1 \\ 9 & \pm 2.66 \\ 5 & \begin{array}{l}41.2 \\ 5\end{array} \pm 2.71\end{array}$

$36.40 \pm 4.6343 .00 \pm 4.37 * * \begin{aligned} & 30.1 \\ & 2\end{aligned} \pm 1.78{ }_{0}^{30.2} \pm 0.97$

$25.10 \pm 3.6518 .16 \pm 2.88 * \begin{aligned} & 16.7 \\ & 6\end{aligned} \pm 1.75 \begin{aligned} & 16.7 \\ & 9\end{aligned} \pm 1.20$ 


\begin{tabular}{|c|c|c|c|c|c|c|c|c|c|c|c|c|c|}
\hline \multirow{3}{*}{ Indicators } & \multicolumn{7}{|l|}{ EG } & \multicolumn{6}{|l|}{ CG } \\
\hline & \multicolumn{3}{|c|}{$\begin{array}{l}\text { Before } \\
\text { experiment }\end{array}$} & \multicolumn{3}{|c|}{$\begin{array}{l}\text { After } \\
\text { experiment }\end{array}$} & \multirow[t]{2}{*}{$\mathbf{P}$} & \multicolumn{3}{|c|}{$\begin{array}{l}\text { Before } \\
\text { experiment }\end{array}$} & \multicolumn{3}{|c|}{$\begin{array}{l}\text { After } \\
\text { experiment }\end{array}$} \\
\hline & M & \pm & m & M & \pm & m & & $\mathbf{M}$ & \pm & m & $\mathbf{M}$ & \pm & m \\
\hline Ruffiet's test (conv.un.) & 10.26 & \pm & 2.07 & 8.64 & \pm & 2.17 & & 8.99 & \pm & 3.66 & 8.81 & \pm & 1.23 \\
\hline Modified Harvard step-test (conv.un.) & 18.90 & \pm & 1.45 & 21.58 & \pm & 1.38 & $* *$ & $\begin{array}{l}19.9 \\
9\end{array}$ & \pm & 0.41 & $\begin{array}{l}21.0 \\
0\end{array}$ & \pm & 1.44 \\
\hline Kerdo's test (conv.un.) & 14.69 & \pm & 3.11 & 10.72 & \pm & 2.61 & $* *$ & $\begin{array}{l}16.7 \\
0\end{array}$ & \pm & 2.74 & $\begin{array}{l}15.8 \\
0\end{array}$ & \pm & 1.51 \\
\hline Skibinskaya's test (conv.un.) & 18.80 & \pm & 3.74 & 29.51 & \pm & 3.70 & $\begin{array}{l}* * \\
*\end{array}$ & $\begin{array}{l}14.0 \\
9\end{array}$ & \pm & 1.53 & $\begin{array}{l}15.0 \\
1\end{array}$ & \pm & 0.76 \\
\hline \multicolumn{14}{|c|}{ Somatic health } \\
\hline Kettle's test of body mass (conv.un.) & 18.00 & \pm & 0.76 & 17.99 & \pm & 0.72 & & $\begin{array}{l}17.2 \\
0\end{array}$ & \pm & 0.23 & $\begin{array}{l}17.2 \\
5\end{array}$ & \pm & 0.33 \\
\hline Vital index (conv.un.) & 47.70 & \pm & 2.58 & 55.94 & \pm & 2.55 & $*$ & $\begin{array}{l}52.0 \\
2\end{array}$ & \pm & 2.14 & $\begin{array}{l}52.5 \\
1\end{array}$ & \pm & 2.52 \\
\hline Power index (conv.un.) & 40.15 & \pm & 2.79 & 38.83 & \pm & 2.17 & & $\begin{array}{l}37.6 \\
9\end{array}$ & \pm & 0.91 & $\begin{array}{l}37.7 \\
5\end{array}$ & \pm & 1.02 \\
\hline $\begin{array}{l}\text { Time of HBR restoration after } 20 \text { squats during } 30 \\
\text { sec. }\end{array}$ & 81.99 & \pm & 6.40 & 65.98 & \pm & 5.99 & & $\begin{array}{l}94.4 \\
1\end{array}$ & \pm & 3.28 & $\begin{array}{l}93.8 \\
9\end{array}$ & \pm & 4.12 \\
\hline Robinson's index (conv.un.) & 93.97 & \pm & 4.74 & 85.78 & \pm & 4.92 & $* *$ & $\begin{array}{l}84.9 \\
8\end{array}$ & \pm & 3.81 & $\begin{array}{l}84.1 \\
1\end{array}$ & \pm & 4.21 \\
\hline General assessment of health level & 4.02 & \pm & 1.34 & 6.77 & \pm & 1.15 & $\begin{array}{l}* * \\
*\end{array}$ & 1.14 & \pm & 0.17 & 1.22 & \pm & 0.32 \\
\hline
\end{tabular}

Notes: *- differences are confident by Student's criteria $(\mathrm{p} \leq 0.05) ; * *$ - differences are confident by Fisher $(p \leq 0.05) ; * * *$ - differences are confident by Student and Fisher $(p \leq 0.05)$.

Table 2. Dynamic of students' indicators in corrected variant of physical culture-health related technology for prophylaxis of respiratory system disorders

\begin{tabular}{|c|c|c|c|c|c|c|c|c|c|c|c|c|c|}
\hline \multirow{3}{*}{ Indicators } & \multicolumn{7}{|l|}{ EG } & \multicolumn{6}{|l|}{ CG } \\
\hline & \multicolumn{3}{|c|}{$\begin{array}{l}\text { Before } \\
\text { experiment }\end{array}$} & \multicolumn{3}{|c|}{$\begin{array}{l}\text { After } \\
\text { experiment }\end{array}$} & \multirow[t]{2}{*}{$\mathbf{P}$} & \multicolumn{3}{|c|}{$\begin{array}{l}\text { Before } \\
\text { experiment }\end{array}$} & \multicolumn{3}{|c|}{$\begin{array}{l}\text { After } \\
\text { experiment }\end{array}$} \\
\hline & $\mathbf{M}$ & \pm & m & $\mathbf{M}$ & \pm & m & & $\mathbf{M}$ & \pm & m & $\mathbf{M}$ & & m \\
\hline \multicolumn{14}{|c|}{ Physical condition } \\
\hline Height, cm & $\begin{array}{l}176.5 \\
4\end{array}$ & \pm & 1.92 & $\begin{array}{l}176.4 \\
9\end{array}$ & \pm & 1.73 & & $\begin{array}{l}178 . \\
79\end{array}$ & \pm & 1.51 & $\begin{array}{l}179 . \\
10\end{array}$ & \pm & 2.37 \\
\hline Weight, kg & 65.54 & \pm & 2.34 & 65.43 & \pm & 2.39 & & $\begin{array}{l}72.6 \\
4\end{array}$ & \pm & 2.10 & $\begin{array}{l}71.0 \\
0\end{array}$ & & 3.51 \\
\hline Weight circumference, cm & 72.43 & \pm & 1.92 & 72.66 & \pm & 1.90 & & $\begin{array}{l}77.1 \\
2\end{array}$ & \pm & 1.48 & $\begin{array}{l}78.2 \\
0\end{array}$ & \pm & 3.39 \\
\hline Excursion of chest, $\mathrm{cm}$ & 7.77 & \pm & 0.58 & 8.85 & \pm & 0.67 & * & 9.32 & \pm & 1.53 & 9.52 & \pm & 0.66 \\
\hline Vital capacity of lungs (VCL), I. & 4.15 & \pm & 0.23 & 4.82 & \pm & 0.16 & $*$ & 4.53 & \pm & 0.13 & 4.53 & \pm & 0.14 \\
\hline Right & 47.99 & \pm & 2.26 & 47.88 & \pm & 2.02 & & $\begin{array}{l}42.2 \\
8\end{array}$ & \pm & 1.26 & $\begin{array}{l}42.8 \\
8\end{array}$ & \pm & 2.66 \\
\hline Hand dynamometry (daN) left & 44.10 & \pm & 2.85 & 44.32 & \pm & 2.61 & & $\begin{array}{l}38.7 \\
5\end{array}$ & \pm & 0.75 & $\begin{array}{l}38.8 \\
9\end{array}$ & \pm & 1.07 \\
\hline average & 46.04 & \pm & 2.51 & 46.10 & \pm & 2.27 & & $\begin{array}{l}40.5 \\
2\end{array}$ & \pm & 1.07 & $\begin{array}{l}41.1 \\
0\end{array}$ & \pm & 2.11 \\
\hline
\end{tabular}




\begin{tabular}{|c|c|c|c|c|c|c|c|c|c|c|}
\hline \multirow{3}{*}{ Indicators } & \multicolumn{5}{|c|}{ EG } & \multicolumn{5}{|c|}{ CG } \\
\hline & \multicolumn{2}{|c|}{$\begin{array}{l}\text { Before } \\
\text { experiment }\end{array}$} & \multicolumn{2}{|c|}{$\begin{array}{l}\text { After } \\
\text { experiment }\end{array}$} & \multirow[t]{2}{*}{$\mathbf{P}$} & \multicolumn{2}{|c|}{$\begin{array}{l}\text { Before } \\
\text { experiment }\end{array}$} & \multicolumn{2}{|c|}{$\begin{array}{l}\text { After } \\
\text { experiment }\end{array}$} & $\mathbf{P}$ \\
\hline & $\mathbf{M}$ & $\pm m$ & $\mathbf{M}$ & $\pm m$ & & $\mathbf{M}$ & $\pm m$ & $\mathbf{M}$ & $\pm m$ & \\
\hline
\end{tabular}

\section{Physical fitness}

Pressing ups in lying position (q-ty of times)

Torso rising from lying position (q-ty of times)

Keeping of half squat with support (sec)

Long jump from the spot $(\mathrm{cm})$

60 meters' run, sec.

10 squatting for quickness, sec.

2000 meters' run for girls and 3000 meters' run for boys, sec.

Bending from bench, $\mathrm{cm}$.

Firiliova's test (conv.un.)

Yarotskiy's test, sec.

Swimming, sec.

Heart beats rate (HBR) in rest, bpm

$\begin{array}{lr}\text { Blood } & \text { pressure } \\ \text { (mm.merc.col.) } & \text { Diastolic }\end{array}$

Systolic blood volume (SBV) (ml)

Minute blood volumes (MBV) (I)

Shtange's test, sec.

Genchy's test, sec.

Orthostatic test (difference bpm)

Ruffiet's test (conv.un.)

Modified Harvard step-test (conv.un.)

Kerdo's test (conv.un.)

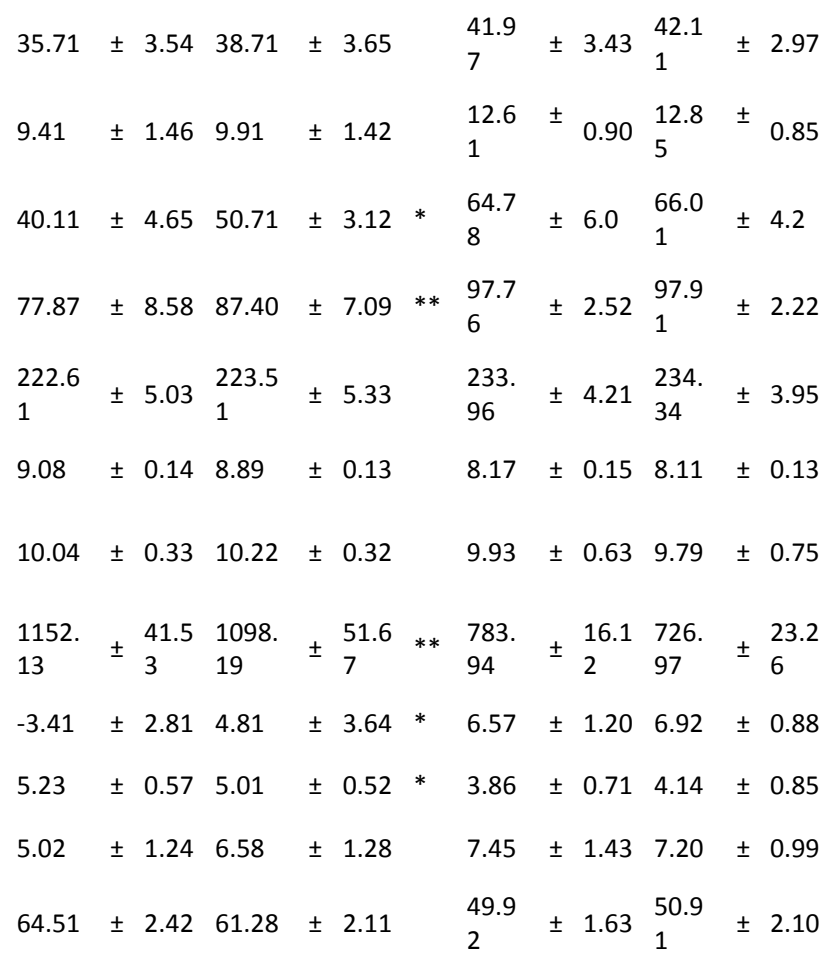

\section{Functional fitness}

\begin{tabular}{|c|c|c|c|c|c|c|c|c|c|c|c|c|}
\hline 4.57 & \pm & 4.05 & 79.02 & \pm & 2.82 & & $\begin{array}{l}75.1 \\
3\end{array}$ & \pm & 4.93 & $\begin{array}{l}77.1 \\
3\end{array}$ & \pm & 5.55 \\
\hline $\begin{array}{l}129.4 \\
6\end{array}$ & \pm & 3.66 & $\begin{array}{l}118.7 \\
9\end{array}$ & \pm & 2.74 & & $\begin{array}{l}130 . \\
63\end{array}$ & \pm & 2.71 & $\begin{array}{l}131 . \\
71\end{array}$ & \pm & 3.11 \\
\hline 70.13 & \pm & 3.12 & 72.06 & \pm & 3.43 & & $\begin{array}{l}73.4 \\
5\end{array}$ & \pm & 0.96 & $\begin{array}{l}72.6 \\
8\end{array}$ & \pm & 1.89 \\
\hline 1.90 & \pm & 4.61 & 63.99 & \pm & 4.53 & & $\begin{array}{l}68.2 \\
2\end{array}$ & \pm & 1.12 & $\begin{array}{l}69.0 \\
6\end{array}$ & \pm & 0.29 \\
\hline 6.00 & \pm & 0.64 & 4.97 & \pm & 0.60 & & 5.07 & \pm & 0.35 & 5.20 & \pm & 0.47 \\
\hline 3.10 & \pm & 4.17 & 77.16 & \pm & 5.01 & $\begin{array}{l}* * \\
*\end{array}$ & $\begin{array}{l}62.4 \\
2\end{array}$ & \pm & 4.24 & $\begin{array}{l}61.0 \\
7\end{array}$ & \pm & 3.77 \\
\hline 39.28 & \pm & 4.61 & 44.10 & \pm & 3.59 & & $\begin{array}{l}39.6 \\
0\end{array}$ & \pm & 4.25 & $\begin{array}{l}37.0 \\
9\end{array}$ & \pm & 3.77 \\
\hline 24.45 & \pm & 2.39 & 20.02 & \pm & 1.85 & $*$ & $\begin{array}{l}19.7 \\
6\end{array}$ & \pm & 1.68 & $\begin{array}{l}20.0 \\
0\end{array}$ & \pm & 0.79 \\
\hline 9.70 & \pm & 1.07 & 8.83 & \pm & 0.56 & & $\begin{array}{l}10.0 \\
3\end{array}$ & \pm & 0.11 & $\begin{array}{l}10.0 \\
2\end{array}$ & \pm & 0.24 \\
\hline 18.26 & \pm & 0.89 & 19.64 & \pm & 0.65 & $*$ & $\begin{array}{l}20.4 \\
4\end{array}$ & \pm & 0.38 & $\begin{array}{l}21.0 \\
9\end{array}$ & \pm & 0.44 \\
\hline 16.96 & \pm & 4.17 & 8.70 & \pm & 3.05 & $\begin{array}{l}* * \\
*\end{array}$ & 6.04 & \pm & 1.65 & 5.98 & \pm & 0.9 \\
\hline
\end{tabular}




\begin{tabular}{|c|c|c|c|c|c|c|c|c|c|c|c|c|c|c|}
\hline \multirow{3}{*}{ Indicators } & & \multicolumn{7}{|l|}{ CG } \\
\hline & \multicolumn{3}{|c|}{$\begin{array}{l}\text { Before } \\
\text { experiment }\end{array}$} & \multicolumn{3}{|c|}{$\begin{array}{l}\text { After } \\
\text { experiment }\end{array}$} & \multirow[t]{2}{*}{$\mathbf{P}$} & \multicolumn{3}{|c|}{$\begin{array}{l}\text { Before } \\
\text { experiment }\end{array}$} & \multicolumn{3}{|c|}{$\begin{array}{l}\text { After } \\
\text { experiment }\end{array}$} & \multirow[t]{2}{*}{$\mathbf{P}$} \\
\hline & $\mathbf{M}$ & \pm & m & $\mathbf{M}$ & \pm & m & & $\mathbf{M}$ & \pm & m & $\mathbf{M}$ & \pm & m & \\
\hline Skibinskaya's test (conv.un.) & 30.13 & \pm & 4.10 & 45.99 & \pm & 3.87 & $\begin{array}{l}* * \\
*\end{array}$ & $\begin{array}{l}35.3 \\
2\end{array}$ & \pm & 3.37 & $\begin{array}{l}37.0 \\
0\end{array}$ & \pm & 2.88 & \\
\hline \multicolumn{15}{|c|}{ Somatic health } \\
\hline Kettle's test of body mass (conv.un.) & 18.62 & \pm & 0.8 & 18.55 & \pm & 0.79 & & $\begin{array}{l}20.2 \\
3\end{array}$ & \pm & 0.42 & $\begin{array}{l}21.0 \\
0\end{array}$ & \pm & 0.13 & $*$ \\
\hline Vital index (conv.un.) & 61.87 & \pm & 3.20 & 72.23 & \pm & 2.98 & & $\begin{array}{l}61.7 \\
9\end{array}$ & \pm & 0.42 & $\begin{array}{l}61.9 \\
0\end{array}$ & \pm & 0.97 & \\
\hline Power index (conv.un.) & 69.95 & \pm & 2.02 & 70.31 & \pm & 1.96 & & $\begin{array}{l}55.8 \\
4\end{array}$ & \pm & 0.18 & $\begin{array}{l}56.9 \\
0\end{array}$ & \pm & 2.24 & \\
\hline $\begin{array}{l}\text { Time of HBR restoration after } 20 \text { squats during } 30 \\
\text { sec. }\end{array}$ & 46.65 & \pm & 5.27 & 43.31 & \pm & 5.26 & & $\begin{array}{l}84.0 \\
7\end{array}$ & \pm & 7.25 & $\begin{array}{l}81.4 \\
9\end{array}$ & \pm & 4.13 & \\
\hline Robinson's index (conv.un.) & $\begin{array}{l}109.6 \\
8\end{array}$ & \pm & 5.46 & 94.05 & \pm & 4.84 & $*$ & $\begin{array}{l}99.9 \\
6\end{array}$ & \pm & 6.59 & $\begin{array}{l}97.8 \\
9\end{array}$ & \pm & 4.81 & \\
\hline General assessment of health level & 6.85 & \pm & 0.92 & 8.72 & \pm & 1.17 & $*$ & 4.60 & \pm & 1.52 & 4.00 & \pm & 0.66 & \\
\hline
\end{tabular}

Experimental technology, in the whole, influenced positively on students' physical fitness. It should be noted that in both groups we found confident increase of general endurance (time of girls' 2000 meters' run and boys' 3000 meters' run reduced). Besides, we registered increment of indicators of shoulder girdle muscles' strength, quickness, flexibility, ability to keep balance. In both groups reduction of 50 meters' distance swimming time was found. It witnesses about complex improvement of physical qualities of the tested. By indicators of functional fitness we also registered positive dynamic. In boys' group confident improvement of cardio-respiratory system general state was determined (by Skibinskaya's index). Not confident improvements of mean values in both groups were found in indicators of organism's aerobic potentials (Shtange's test), vegetative indicators (orthostatic test and Kerdo's index) and physical workability (step-test).

Calculation of somatic health indices proved experimental technology's general positive influence on health of the tested. In both groups we registered improvement of vital index, Robinson's index; time of heart beats rate restoration after moderate physical load mean values. All mentioned indicators are important characteristics of respiratory system's condition. Total points of somatic health in both groups increased (though not confidently) but remained within level "below average".

Pilot testing of new technology showed its general positive influence on students' somatic health indicators. Alongside with it, initial variant of the technology did not render the expected effect on the following indicators: vital capacity of lungs, chest excursion, time of breathing pause in tests of Genchy and Shtange.

In our opinion insufficient effectiveness of experimental technology can be connected with little quantity of aerobic exercises, absence of everyday hardening procedures, partial mastering of recommended complexes of breathing exercises, limited time for technology's testing. In this connection we inserted the following corrections in initial variant of physical culture-health related technology for prophylaxis of respiratory disorders:

- Health walks as part of students' everyday independent trainings;

- If no counter indications, - everyday health swimming;

- Every evening hardening procedures;

- All exercises of breathing complexes.

Effectiveness of the mentioned corrections was proved in the course of pedagogic experiment. Analysis and generalization of the received data witness about the following:

- The corrected variant of the technology, in the whole, rendered positive influence on students' physical condition - in both groups indicators of chest excursion and vital capacity of lungs increased confidently;

- This variant of the technology also positively influenced on students' physical fitness: in both groups we noted confident improvement of abdomen muscles' strength and flexibility; in girls' group - complex 
manifestation of all physical qualities; in boys' group - static strength of legs' muscles, endurance, motor coordination;

- The most noticeable effect was rendered on students' functional fitness: in both groups organism's aerobic potentials confidently increased as well as physical workability; vegetative indicators and general state of cardio-respiratory system; in girls' group organism's resistance to hypoxia confidently improved. Besides, in both groups we registered optimization of myocardium functioning and blood pressure stabilization;

- Students' general level of somatic health confidently increased. It permitted to rise points of boys' group from "below average" to "average"; in girls' group - somatic health level remained on level "below average".

Analysis and generalization of control groups' students witness about the following:

- Physical condition did not change noticeably during semester cycle of trainings: in both groups we registered confident improvement of right hand dynamometry, while there were no confident changes in the rest of indicators;

- Physical fitness improved to some extent: in girls group we noticed confident improvement of explosive power and motor coordination; in boys' group abdomen muscles' strength and flexibility confidently increased;

- Functional fitness also improved a little: in both groups general; level of cardio-respiratory system's functioning increased; in boys' group physical workability improved;

- General level of somatic health improved confidently that permitted to change girls' group points from "low" level to level "below average"; in boys" group the calculated health level remained on level "below average".

Thus, results of control section of somatic health indicators of control groups' students witness about general positive influence of academic program on the tested parameters. We can conclude that this program, in general, realized the envisaged by it functions - health related and developing. Alongside with it, the worked out by us physical culture-health related technology for prophylaxis of respiratory system disorders is more effective, comparing with academic program.

\section{Discussion}

The fulfilled by us theoretical analysis and generalization of literature data on prophylaxis of students' respiratory system disorders prove the presence of problem situation [15, 20, 21, 22, 23].

At present time, for Russian HEE physical education system the tasks of somatic health improvement and students' motor functioning; cultivation of students' demand in systemic physical culture practicing (including independent); effective means of attractive for students kinds of motor functioning implementation in curriculum in students are the most urgent.

In our opinion all these prove relevance of our research and witness about demand in working out of new physical culture-health related technologies and their implementation in educational space of modern HEE. Such technologies shall facilitate development and perfection of basic physical qualities, formation of main motor skills, health strengthening, provisioning of optimal conditions for organism's functional systems work. Such approach permits to keep student's mental and physical workability at high level.

The tendency to students' health worsening actualizes demand in working out modern physical culturehealth related technologies and their implementation in HEE educational space. Such technology shall be based on complex application of medical-biological and psychological-pedagogical means. Besides, means of motor functioning, having systemic-selective character, shall be in its basis $[1,2,3,4,5,18]$.

\section{Conclusions}

Thus, the worked out by us physical culture-health related technology for prophylaxis of respiratory system disorders is the main result of our research. This technology has been built on the base of synthesis of the most effective health related physical culture means. In its base we put generalized data about their targeted influence. In this connection the main characteristic of our physical culture-health related technology is its orientation on solution of definite health improvement tasks, according to peculiarities of trainees' contingent. In particular, this technology permits to present its content and orientation as an integral mean of improvement of organism's functional potentials, as well as mean of students' educational process effectiveness rising. It permits to significantly reduce after effects of students' respiratory system disorders.

\section{Conflict of interests}

The authors declare that there is no conflict of interests.

\section{References}

1. Balysheva NV. Ukreplenie zdorov'ia studentok, imeiushchikh narusheniia serdechno-sosudistoj sistemy, sredstvami dozirovannoj ozdorovitel'noj khod'by i bega. Cand. Diss. [Strengthening of girl students' health, 
who have cardio-vascular system disorder, by means of dozed health walks and run. Cand. Diss.], Sankt Petersburg; 2010.

2. Bogoeva MD, Rumba OG, Gorelov AA. Postroenie processa fizicheskogo vospitaniia studentov special'noj medicinskoj gruppy s ogranichennymi vozmozhnostiami serdechno-sosudistoj sistemy [Building of physical education process for special health groups' students with cardio-vascular system problems], Belgorod: Politerra; 2011.

3. Butenko MV. Formirovanie kul'tury ZOZh lichnosti studenta v processe zaniatij atleticheskoj gimnastikoj. Cand. Diss. [Formation of HLS culture of student's personality in process of athletic gymnastic practicing. Cand. Diss.], Omsk: SibGUFK; 2004.

4. Vinogradov IG. Soderzhanie rekreacionnykh zaniatij atletizmom so studentami vuzov. Cand. Diss. [Content of athletic recreational trainings of HEE students. Cand. Diss.], Sankt Petersburg; 2008.

5. Gorelov AA, Rumba OG, Kopejkina EN. Dykhatel'nye uprazhneniia kak faktor uluchsheniia sostoianiia zdorov'ia studentov s zabolevaniiami organov dykhaniia [Breathing exercises as factor of health improvement of students with respiratory organs' disorders]. Uchenye zapiski universiteta im. P.F. Lesgafta, 2008;11(45):21 - 25 .

6. Kovaleva MV, Rumba OG. Primenenie podvizhnykh i elementov sportivnykh igr na zaniatiiakh so studentami s ogranichennymi vozmozhnostiami zdorov'ia serdechno-sosudistoj sistemy [Application of outdoor games and elements of sports games at trainings of students with cardio-vascular system problems], Belgorod: Politerra; 2012.

7. Kondakov VL, Gorelov AA. Novyj vzgliad na mekhanizmy konstruirovaniia fizkul'turno-ozdorovitel'nykh tekhnologij v obrazovatel'nom prostranstve sovremennogo vuza [New view at mechanisms of physical culture-health related technologies' construction in educational space of modern HEE]. Nauchnye Vedomosti Belgorodskogo gosudarstvennogo universiteta, 2014;13(22):273-282.

8. Kondakov VL. Sistemnye mekhanizmy konstruirovaniia fizkul'turno-ozdorovitel'nykh tekhnologij $v$ obrazovatel'nom prostranstve sovremennogo vuza. Doct. Diss. [System mechanisms of physical culturehealth related technologies' construction in educational space of modern HEE. Doct. Diss.], Sankt Petersburg; 2013.

9. Kopejkina EN, Bogoeva MD. Dykhatel'nye uprazhneniia kak sredstvo povysheniia somaticheskogo zdorov'ia studentov [Breathing exercises as mean of students' somatic health strengthening]. Ekonomicheskie $i$ gumanitarnye issledovaniia regionov, 2011;6:17-23.

10. Kopejkina EN. Postroenie processa fizicheskogo vospitaniia studentok s narusheniiami v sostoianii dykhatel'noj sistemy. Cand. Diss. [Building of physical education process for girl students with respiratory system disorders. Cand. Diss.], Sankt Petersburg; 2010.

11. Postanovlenie pravitel'stva RF ot 28 iiulia 2008g. vol. 568 “O federal'noj celevoj programme "Nauchnye $i$ nauchno-pedagogicheskie kadry innovacionnoj Rossi» na 2009-2013 gody” [Decree of RF government No. 568, dt. July 28, 2008 "On federal targeted program "Scientific and pedagogic specialists of innovative Russia” for 2009-2013”]. [Online] Available from: http://base.garant.ru/6390825/\#ixzz447GSmq9q [Accessed 15th April 2016].

12. WMA Declaration of Helsinki - Ethical Principles for Medical Research Involving Human Subjects. [Online] Available from: [Accessed 15th April 2016]. http://www.ub.edu/recerca/Bioetica/doc/Declaracio_Helsinki_2013.pdf

13. Chen Q, Shi M-Y, Zhang W, Tian J. Effect of breathing and Daoyin exercises on the quality of life in patients with chronic obstructive pulmonary disease. Journal of Acupuncture and Tuina Science, 2015;3(6):353-360.

14. Drogomeretsky VV, Kondakov VL, Gorelov AA. Application of improving swimming to the correction of joint and ligament students. Physical Education of Students, 2013;5:46-54. doi:10.6084/m9.figshare.771046

15. Drăgoi R-G, Amaricai E, Drăgoi M, Popoviciu H, Avram C. Inspiratory muscle training improves aerobic capacity and pulmonary function in patients with ankylosing spondylitis: A randomized controlled study. Clinical Rehabilitation, 2016;30(4):340-346.

16. Gorelov AA, Obvintsev AA, Kondakov VL. Design and functioning of health and fitness technologies in educational environment of military educational institution. Theory and practice of physical culture, 2014;9:210 .

17. Kondakov VL, Kopeikina EN, Balysheva NV, Usatov AN, Skrug DA. Causes of declining interest of students to employment physical education and sports. Physical education of students, 2015;1:22-30. http://dx.doi.org/10.15561/20755279.2015.0104

18. Kondakov VL, Voloshina LN, Balysheva NV, Kopeikina EN, Skrug DA. Correction of the state of cardiovascular system of undergraduates by means of dosed constitutional walking and jogging. Research Journal of Medical Sciences, 2015;9(3):95-98. 
19. Kondakov VL, Kopeykina EN, Balysheva NV. Health and fitness technology to prevent respiratory disorders. Theory and practice of physical culture, 2016;1:34-36.

20. Martín-Salvador A, Colodro-Amores G, Torres-Sánchez I, Cabrera-Martos I, Valenza MC. Intervención fisioterápica durante la hospitalización en pacientes con exacerbación aguda de la enfermedad pulmonar obstructiva crónica y neumonía: un ensayo clínico aleatorizado [Physical therapy intervention during hospitalization in patients with acute exacerbation of chronic obstructive pulmonary disease and pneumonia: A randomized clinical trial]. Medicina Clinica, 2016;146(7):301-304.

21. Mullur LM, Khodnapur J, Bagali S, Dhanakshirur GB, Aithala M. A study of short term yoga training effect on respiratory endurance and muscle strength in elderly individuals. Indian Journal of Public Health Research and Development, 2014;5(4):37-40.

22. Syropoulos S, Kalogeropoulos A, Maniatis G, Iliodromiti Z. The effect of respiratory physiotherapy on pain and pulmonary complications in major upper abdominal surgery. Archives of Hellenic Medicine, 2016;32(6):724-731.

23. Wanrooij VHM, Willeboordse M, Dompeling E, Van De Kant KDG. Exercise training in children with asthma: A systematic review. British Journal of Sports Medicine, 2014;48(13):1024-1031.

\begin{tabular}{|c|}
\hline Information about the authors: \\
\hline $\begin{array}{l}\text { Kondakov V.L.; http://orcid.org/0000-0002-8094-0144; } \\
\text { kondakov@bsu.edu.ru; National Research University Belgorod } \\
\text { State University; Pobeda 85, Belgorod, 308015, Russia. }\end{array}$ \\
\hline $\begin{array}{l}\text { Kopeikina E.N.; http://orcid.org/0000-0002-6027-3495; } \\
\text { kopeikina@bsu.edu.ru; National Research University Belgorod } \\
\text { State University; Pobeda 85, Belgorod, 308015, Russia. }\end{array}$ \\
\hline $\begin{array}{l}\text { Usatov A.N.; } \quad \text { http://orcid.org/0000-0002-9148-5060; } \\
\text { usatov@bsu.edu.ru; National Research University Belgorod } \\
\text { State University; Pobeda 85, Belgorod, 308015, Russia. }\end{array}$ \\
\hline $\begin{array}{l}\text { Cite this article as: Kondakov V.L., Kopeikina E.N., Usatov } \\
\text { A.N. On purposefulness of application of physical culture- } \\
\text { health related technology for prophylaxis of students' } \\
\text { respiratory system disorders. Physical education of students, } \\
\text { 2016;2:4-12. doi:10.15561/20755279.2016.0201 }\end{array}$ \\
\hline 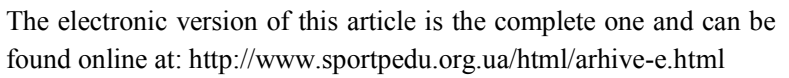 \\
\hline $\begin{array}{l}\text { This is an Open Access article distributed under the terms of the } \\
\text { Creative Commons Attribution License, which permits unrestricted } \\
\text { use, distribution, and reproduction in any medium, provided the } \\
\text { original } \quad \text { work } \quad \text { is } \quad \text { properly } \\
\text { (http://creativecommons.org/licenses/by/4.0/deed.en). }\end{array}$ \\
\hline $\begin{array}{l}\text { Received: } 03.03 .2016 \\
\text { Accepted: } 19.03 .2016 \text {; Published: } 25.04 .2016\end{array}$ \\
\hline
\end{tabular}

